\title{
Enantioselective Oxidative Damage of Chiral Pesticide Dichlorprop to Maize
}

\author{
Tong Wu, Xiuying Li, Honglin Huang, and Shuzhen Zhang* \\ State Key Laboratory of Environmental Chemistry and Ecotoxicology, Research Center for Eco-Environmental Sciences, \\ Chinese Academy of Sciences, Beijing 100085, People's Republic of China
}

ABSTRACT: To investigate the enantioselective oxidative damage of the pesticide dichlorprop (DCPP) to maize, young seedlings were exposed to solutions of DCPP enantiomers and racemate at different concentrations. Early root development was more influenced by $(R)$-DCPP than racemic $(\mathrm{rac})$ - and $(S)$-DCPP. Inhibition rates of seed germination, seedling biomass, and root and shoot elongation were all in the order of $(R)$-DCPP $>(\mathrm{rac})$-DCPP $>(S)$-DCPP treatments. The antioxidant enzyme activities of superoxide dismutase (SOD) and peroxidase (POD) were significantly upregulated by exposure to lower concentrations of $(R)$ DCPP than (rac)- and (S)-DCPP. Direct determination of the formation of hydroxyl radical $(\bullet \mathrm{OH})$ with electron paramagnetic resonance (EPR) spectroscopy indicated that the $\bullet \mathrm{OH}$ level in maize roots followed the order of $(R)$-DCPP $>(\mathrm{rac})$-DCPP $>(S)$ DCPP treatments. All of these results provide solicited evidence of the significant enantioselective phytotoxicity of DCPP to maize with a higher toxicity of $(R)$-DCPP than $(S)$ - and ( rac)-DCPP.

KEYWORDS: Oxidative damage, enantioselective phytotoxicity, dichlorprop (DCPP), hydroxyl radical

\section{INTRODUCTION}

Many modern pesticides are chiral compounds and consist of two or more enantiomers. Chiral enantiomers have identical physical and chemical properties in achiral environments (e.g., air-water exchange, sorption, and abiotic transformation), but they show different activities in biological systems because individual enantiomers can interact enantioselectively with enzymes and biological receptors in organisms. ${ }^{1,2}$ Enantioselectivity of chiral pesticides in the environment, such as enantioselective degradation and toxicity, is receiving increasing attention. 3

Despite their application to soil and direct exposure to plants, enantioselective toxicity of chiral pesticides to plants has not received enough attention. ${ }^{4}$ Only a limited amount of research has been performed on toxicity of chiral pesticides to plants at the chiral level. To our knowledge, all of the available information related to enantioselective toxicities of chiral pesticides include research on the chemicals of paclobutrazol, ${ }^{5} 1$-( $\alpha$-methylbenzyl)-3-( $p$-tolyl)urea, ${ }^{6-9}$ diclofop, ${ }^{10}$ and imazethapyr. ${ }^{11}$ These studies demonstrated significant enantioselective toxicity of the chiral pesticides to plants. However, such a conclusion was drawn in most of these studies solely based on the different influence of enantiomers on the inhabitation of plant growth, with the exception of the research by Qian et al. ${ }^{11}$ and Ye et al., ${ }^{12}$ which evolved enantioselective effects of enantiomers on antioxidant enzyme activity and the chloroplast Hill reaction. Recently, Liu et al. ${ }^{4}$ did an excellent review of the research in phytotoxicity of chiral plant growth regulators and chiral herbicides, in which they particularly emphasized the importance of the issues regarding the interactions between chiral compounds and plants.

When plants are exposed to contaminants, such as pesticides, they often suffer oxidative stress caused by the generation of reactive oxygen species (ROS), such as superoxide anion radical $\left(\mathrm{O}_{2}{ }^{\bullet}\right)$, hydroxyl radical $(\bullet \mathrm{OH})$, singlet oxygen $\left({ }^{1} \mathrm{O}_{2}\right)$, and hydrogen peroxide $\left(\mathrm{H}_{2} \mathrm{O}_{2}\right) .{ }^{13}$ Plants have evolved a variety of mechanisms to deal with the damaging effects of ROS in cellular compartments, which are generally handled through the production of various antioxidative enzymes, such as superoxide dismutase (SOD) and peroxidase (POD). SOD can catalyze superoxide radicals $\left(\mathrm{O}_{2}{ }^{-}\right)$to $\mathrm{H}_{2} \mathrm{O}_{2}$ and $\mathrm{O}_{2}{ }^{14}$ and POD is mainly responsible for hydrolysis of $\mathrm{H}_{2} \mathrm{O}_{2} \cdot{ }^{15}$ Thus, measuring these enzymes can serve as a method to evaluate plant oxidative stress. ${ }^{16-18}$ Nevertheless, hardly any research has been performed to directly determine ROS generation in plants subjected to oxidative stress.

Dichlorprop (DCPP), widely used to control annual grasses and certain broadleaf weeds, exists as a single pair of enantiomers with $(S)$ and $(R)$ configurations. It belongs to systemic herbicides and mimics the action of plant growth hormones (auxins). DCPP has a relatively high water solubility and persistence in the environment; ${ }^{19}$ therefore, application can cause its accumulation in soil and damage to plants. Enantiomers of DCPP may have different phytotoxicity; however, this remains unclear.

The aim of the present study was therefore to investigate the enantioselective toxicity of DCPP in maize. Effects of different concentrations of DCPP enantiomers and racemate on root development, seed germination, seedling biomass, and root and shoot elongation were investigated. To achieve a better understanding of the mechanisms involved, antioxidant enzymes of SOD and POD were measured and ROS generation in maize was detected by electron paramagnetic resonance (EPR) spectroscopy. All of these parameters were compared between treatments of racemic ( $\mathrm{rac})_{-},-(S)$-, and $(R)$-DCPP to assess the toxicity of different enantiomers.

Received: January 16, 2011

Revised: February 25, 2011

Accepted: February 28, 2011

Published: March 14, 2011 


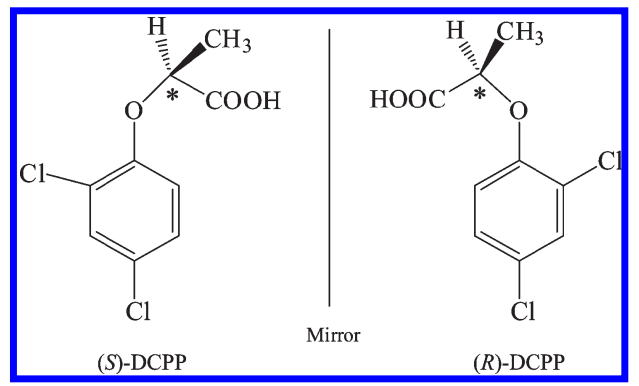

Figure 1. Chemical structures of DCPP enantiomers.

\section{MATERIALS AND METHODS}

Chemicals and Materials. Analytical standards of ( $\mathrm{rac}$ )- and $(R)$ DCPP with a purity of 98.1 and $98.5 \%$ were purchased from AccuStandard (New Haven, CT). The stereoconfigurations of DCPP are shown in Figure 1. Dimethyl sulfoxide (DMSO, purity $>99.9 \%$ ) and $\alpha$-phenyl- $N$-tert-butylnitrone (PBN, purity $>98 \%$ ) were purchased from Sigma-Aldrich Company (St. Louis, MO). Other solvents and chemicals used were of analytical or high-performance liquid chromatography (HPLC) grade.

Preparation and Identification of Enantiomers. Enantiomeric separation was performed on HPLC (Agilent 1100 series) equipped with a G1315A diode array detector (DAD). The HPLC column used for separation was a Chirobiotic T column (Sigma-Aldrich Co., St. Louis, MO; $250 \times 4.6 \mathrm{~mm}$ inner diameter; $5 \mu \mathrm{m}$ particle size), protected with a guard column of the same phase. Separation of the enantiomers was delivered at $0.9 \mathrm{~mL} \mathrm{~min}{ }^{-1}$ and consisted of 20:80 methanol/1\% triethylammonium acetate ( $\mathrm{pH} 4.1)$, with $240 \mathrm{~nm}$ of detection wavelength. ${ }^{20}$ All of the solvents were filtered through a 0.45 $\mu \mathrm{m}$ membrance filter and sonicated prior to use. Under the optimal conditions, a baseline separation $\left(R_{\mathrm{s}}=1.5\right)$ was achieved for DCPP enantiomers.

Single enantiomers were prepared by manually collecting the eluents corresponding to the resolved peaks at the HPLC outlet of the detector while observing the UV signals. The collected samples were then evaporated to dryness, redissolved in methanol, and used as the stock solutions for bioassays (the final amount of methanol in the assay solution was $<0.05 \%$ ). The enantiopurities of the prepared enantiomers were $>98 \%$, checked under the HPLC conditions.

$(\mathrm{rac})$ - and (R)-DCPP were injected into HPLC to ascertain the optical rotation of each enantiomer. Because the same enantiomer at a chiral column has the same retention time, the absolute configurations of enantiomers were confirmed. The first eluted peak was the $(S)$ enantiomer, and the second eluted peak was the $(R)$ enantiomer, which is consistent with the results by Schneiderheinze et al. ${ }^{20}$

Seed Germination and Toxicity Assay. Effects of different concentrations of (rac)-, $(S)$-, and (R)-DCPP on seed germination and toxicity assay were evaluated. A series of (rac)-, $(S)$-, and (R)-DCPP solutions with a range of concentrations $(0,0.02,0.05,0.1,0.5$, and 1.0 $\mathrm{mg} \mathrm{L} \mathrm{L}^{-1}$ ) were prepared as the test solutions using sterile deionized water.

Maize (Zea mays L.) seeds were obtained from the Chinese Academy of Agricultural Sciences, Beijing, China. Prior to germination, the seeds were surface-sterilized in $3 \%(\mathrm{v} / \mathrm{v}) \mathrm{H}_{2} \mathrm{O}_{2}$ for $10 \mathrm{~min}$ and rinsed thoroughly with deionized water. Seeds were germinated on filter paper placed in Petri dishes and moistened by deionized water at $27 \pm 0.5^{\circ} \mathrm{C}$ in the dark. After 4 days, five uniformly germinated seedlings were transferred to each colored vitreous pot containing $150 \mathrm{~mL}$ of test solution. Each treatment was set up in triplicate and repeated 3 times. Pots were kept in a controlled environment growth chamber at a light intensity of $250 \mu \mathrm{mol} \mathrm{m} \mathrm{s}^{-2}$ provided by supplementary illumination with a photoperiod of $14 \mathrm{~h}$ each day, at a $25 / 20{ }^{\circ} \mathrm{C}$ day/night temperature regime and a relative humidity of $80 \%$. The seedlings were repositioned daily to minimize spatial differences in illumination and temperature. The inhibition of fresh weight, root length, and shoot height compared to the control was determined after 3 days of exposure. Test solutions were renewed every day to avoid change in the concentration and enantioselective transformation. DCPP concentrations in the test solutions after the exposure experiment were analyzed, and the changes were less than $8 \%$.

Seed germination was tested on filter paper placed in Petri dishes and moistened with $15 \mathrm{~mL}$ of test solutions of DCPP at concentrations of $0.02,0.05,0.1,0.5$, and $1.0 \mathrm{mg} \mathrm{L}^{-1}$, respectively. Controls were obtained by moistening the filter paper with $15 \mathrm{~mL}$ of deionized water. A total of 100 seeds after sterilizing were placed in each dish, covered by a lid, and incubated at $27 \pm 0.5^{\circ} \mathrm{C}$ in the dark. The test solutions were renewed every day, and the proportion of seeds that had germinated after 3 days was counted. Seeds were considered to have germinated when both the radicle and coleoptile length were over half of the seed size. Each treatment was set up in triplicate and repeated 3 times.

Enzyme Extraction and Analysis. Seedlings after 3 days of exposure were rinsed thoroughly with distilled water, blotted with tissue paper, and separated into roots and shoots. Fresh roots $(1.0 \mathrm{~g})$ were homogenized in $10 \mathrm{~mL}$ of extraction buffer $\left[0.05 \mathrm{M} \mathrm{NaH}_{2} \mathrm{PO}_{4} \cdot \mathrm{Na}_{2} \mathrm{H}\right.$ $\mathrm{PO}_{4}$ in $1 \%(\mathrm{w} / \mathrm{v})$ polyvinylpyrrolidone at $\left.\mathrm{pH} 7.8\right]$ in an ice bath. The supernatant from the centrifugation at $10000 \mathrm{rpm}$ for $10 \mathrm{~min}$ at $4{ }^{\circ} \mathrm{C}$ was used for further analyses. The protein level was measured by the method from Bradford, ${ }^{21}$ using bovine serum albumin as the standard. The determination of SOD and POD followed the same principles as that by Beauchamp and Fridovich and Lagrimini. ${ }^{22,23}$

PBN Spin Trapping and EPR Analysis. Maize roots after 3 days of exposure to 0.05 and $1.0 \mathrm{mg} \mathrm{L}^{-1}(\mathrm{rac})$-, $(S)$-, and (R)-DCPP solutions were subjected to detection of ROS generated. The PBN radical adduct in plant samples was determined according to Shi et al., ${ }^{24}$ with some modifications. The operations were conducted in nitrogen. After the plant samples were rinsed with ice-cold $0.1 \mathrm{M} \mathrm{CaCl}_{2}$ solution, they were weighed $(0.5 \mathrm{~g})$ and homogenized in a mortar with $1.0 \mathrm{~mL}$ of freshly prepared solution of $100 \mathrm{mM}$ PBN (dissolved in DMSO). Then, the homogenates were incubated at $37{ }^{\circ} \mathrm{C}$ for $15 \mathrm{~min}$ and centrifuged at $4500 \mathrm{rpm}$ for $3 \mathrm{~min}$ at $4{ }^{\circ} \mathrm{C}$, and the supernatant was collected. A portion of $30 \mu \mathrm{L}$ of supernatant was transferred to a capillary tube with a diameter of $1.0 \mathrm{~mm}$ for EPR analysis.

The EPR spectra were recorded with a Bruker ESP 300 spectrometer (Bruker, Germany) at room temperature. The operation conditions are as follows: microwave power, $20 \mathrm{~mW}$; microwave frequency, $9.7 \mathrm{GHz}$; center field, $347 \mathrm{mT}$; scan range, $10 \mathrm{mT}$; modulation frequency, 100 $\mathrm{kHz}$; modulation amplitude, $0.25 \mathrm{mT}$; and receiver gain, $2 \times 10^{4}$ scans. The intensities of free radicals were calculated on the basis of the central peak heights of EPR signals.

Statistical Analysis. All results were expressed as an average of three replications. Statistical analysis was performed by Microsoft Excel, Origin 7.0, and SPSS 11.0 variance analysis software. The statistical significance was set at $p<0.05$.

\section{RESULTS AND DISCUSSION}

Enantioselective Effects of DCPP on Maize Growth. Morphological differences of maize roots existed among enantiomer treatments, as shown by the photographs in Figure 2, by taking $0.1 \mathrm{mg} \mathrm{L}^{-1}$ DCPP treatment as an example. The most significant observation was that maize treated with $(S)$-DCPP had much longer and denser root hairs than all of the other treatments, whereas $(R)$-DCPP treatment inhibited root hair development. This observation is consistent with the previous report by Qian et al., ${ }^{11}$ in which they indicated that $(R)$-imazethapyr markedly 


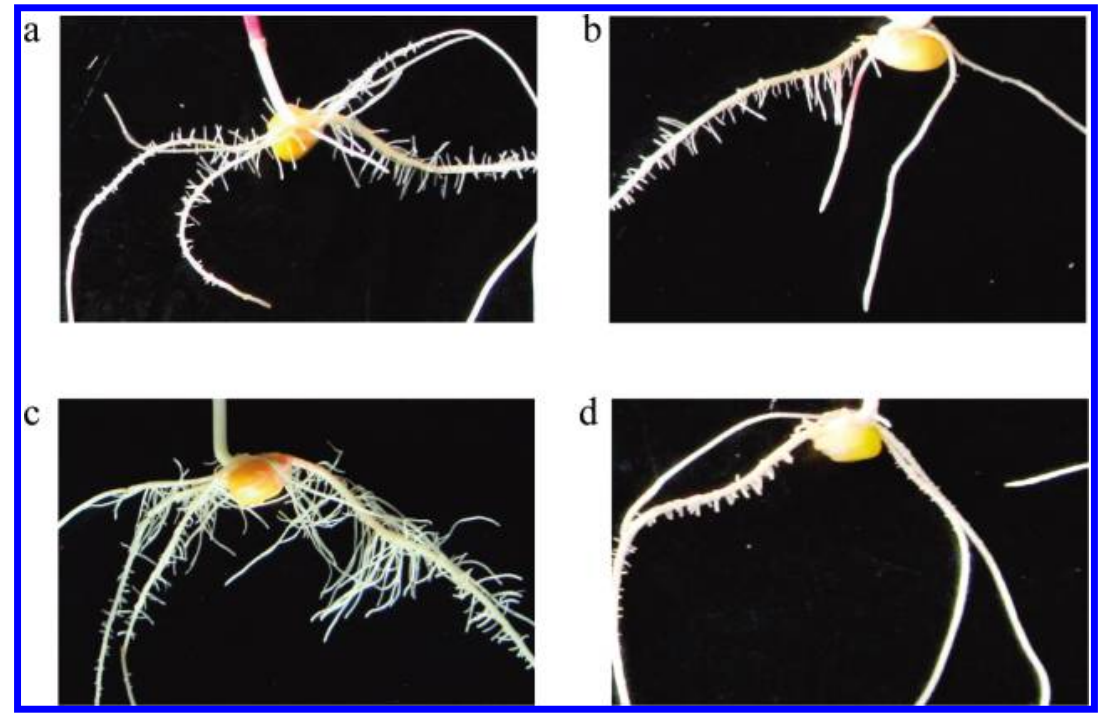

Figure 2. Photos of maize roots treated with $0.1 \mathrm{mg} \mathrm{L}^{-1}(\mathrm{rac})$-, $(S)$-, and (R)-DCPP for 3 days: (a) control, (b) (rac)-DCPP treatment, (c) (S)-DCPP treatment, and (d) (R)-DCPP treatment.

Table 1. Relative Inhibition Rate of Germination, Fresh Weight, and Root and Shoot Elongation of Maize Exposed to DCPP Racemate and Enantiomers at $0.02,0.05,0.1,0.5$, and $1.0 \mathrm{mg} \mathrm{L}^{-1}$ after 3 Days of Exposure

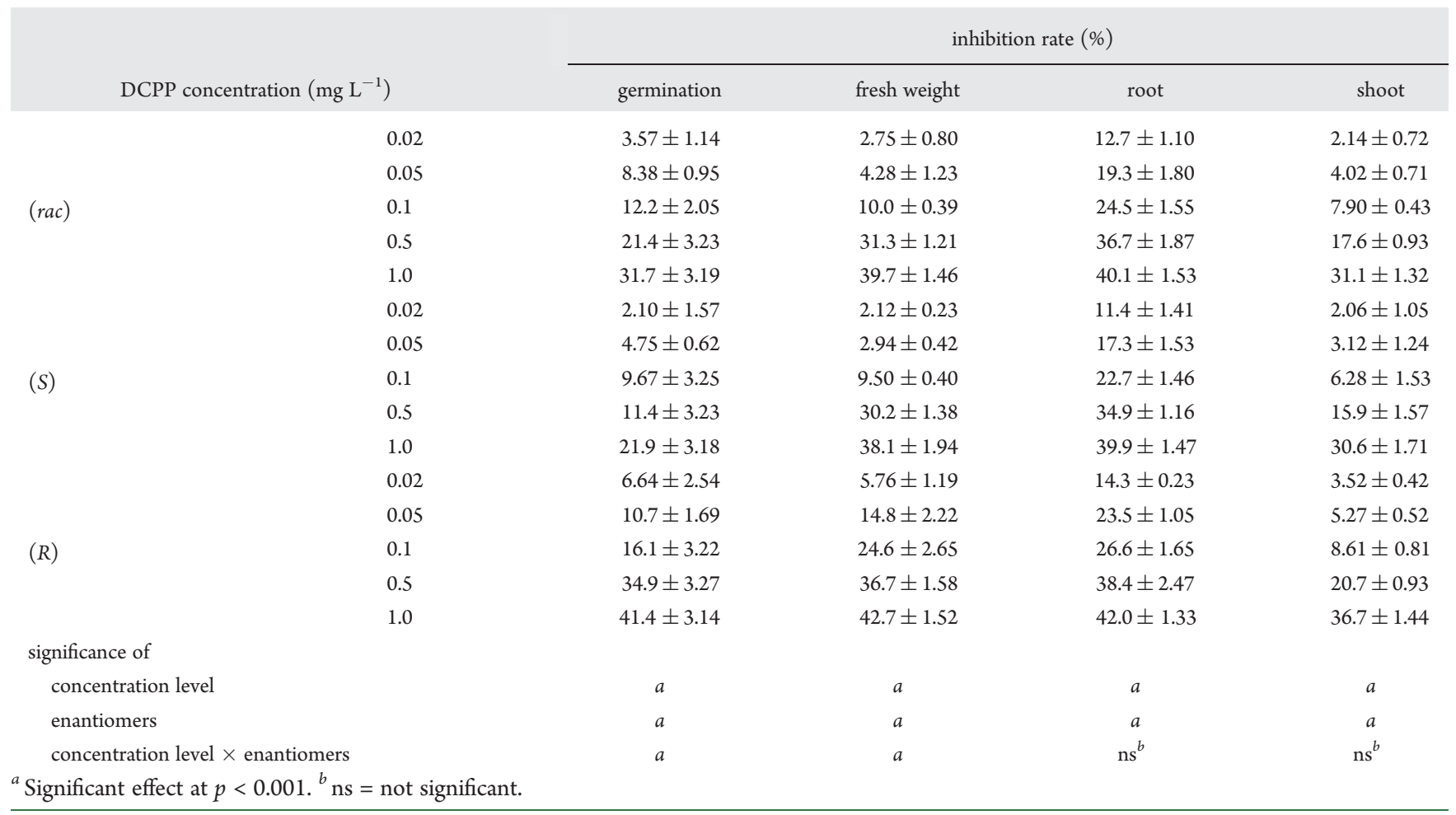

inhibited the growth of primary roots, adventitious roots, and root hairs of rice compared to $(S)$ - and $(\mathrm{rac})$-imazethapyr. Root hairs are subcellular extensions from the root epidermis and can contribute up to $67 \%$ of the total root surface area, which contributes to the acquisition of nutrients. Therefore, dense root hairs can facilitate plant uptake of nutrients, benefiting plant growth, whereas sparse root hairs will reduce nutrient uptake and cause plant damage. ${ }^{25}$

Inhibition rates on seed germination, seedling biomass, and root and shoot elongation were investigated, and they increased significantly with an increasing exposure concentration of DCPP, irrespective of the chiral configuration (Table 1). All of the inhibition indexes are in the following order: $(R)$-DCPP $>(\mathrm{rac})$ DCPP > (S)-DCPP treatments. Taking $0.1 \mathrm{mg} \mathrm{L}^{-1} \mathrm{DCPP}$ treatment as an example, the inhibition rate of seedling biomass for (R)-DCPP treatment was 24.6\%, 2.6 and 2.5 times higher than $(S)$ - and ( rac)-DCPP treatments, respectively. Recently, Zhou et al. ${ }^{26}$ have reported a similar trend for imazethapyr, with a more severe influence of the $(R)$ enantiomer on maize root development than that of the $(S)$ enantiomer. In comparison, the 


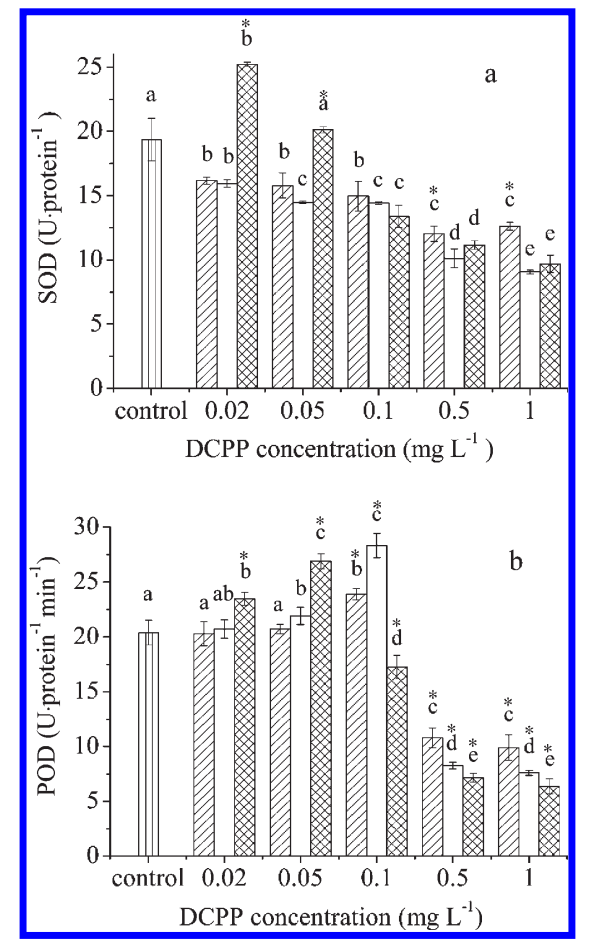

Figure 3. Activities of (a) SOD and (b) POD in maize roots exposed to DCPP enantiomers from 0 to $1.0 \mathrm{mg} \mathrm{L}^{-1}$ for 3 days. Each value is the mean \pm standard error of the mean (SEM) of three replicate cultures. Different letters represent a statistically significant difference between different concentrations at $p<0.05$, while $*$ represents a significant difference between the enantiomers at $p<0.05$ (diagonal line bars, $(\mathrm{rac})$ DCPP; open bars, $(S)$-DCPP; crossed line bars, $(R)$-DCPP; and vertical line bars, control).

inhibition rates of root elongation were the highest for all of the treatments, irrespective of the chiral configuration and concentration of DCPP, with one exception of $1.0 \mathrm{mg} \mathrm{L}^{-1}(R)$ enantiomer treatment (Table 1), suggesting roots were the most sensitive to DCPP toxicity. These observations of enantioselective inhibition of seed germination, root development, and seeding growth evidently demonstrate the significant enantioselective toxicity of DCPP to maize, and the toxicity of $(R)$-DCPP was much higher than that of $(\mathrm{rac})$ - and $(S)$-DCPP.

Enantioselective Effects on Antioxidative Enzyme Activities. Oxidative stress, resulting from the deleterious effects of ROS, is an important phenomenon in plants when exposed to contamination. ${ }^{27}$ Nevertheless, very limited studies have investigated enantioselective effects of chiral pesticides on plant oxidative damage. Antioxidative enzymes of SOD and POD in plants play important roles in scavenging ROS produced under oxidative stress. $^{28}$ Therefore, the changes of these enzyme activities can be considered as circumstantial evidence for enhanced production of ROS.

The SOD activity decreased gradually with an increasing concentration of (rac)- and (S)-DCPP, whereas for (R)-DCPP treatment, it was significantly increased at a concentration of 0.02 $\mathrm{mg} \mathrm{L}^{-1}$ and then decreased (Figure $3 \mathrm{a}$ ). This suggests that the oxidative damage was obvious for the exposure of $(R)$-DCPP but not obvious for the exposures of ( $r a c)$ - and $(S)$-DCPP. The results in Figure $3 \mathrm{~b}$ showed that the POD activity increased at first with an increasing exposure concentration of all of the DCPP enantiomers and then decreased. However, the concentration

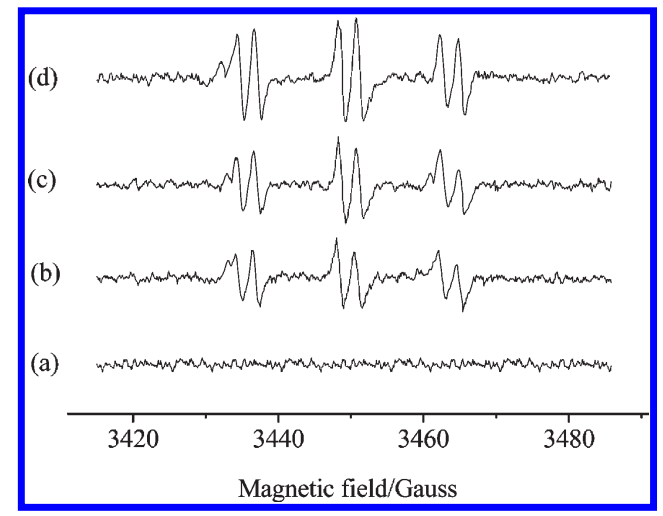

Figure 4. EPR spectrum of $\mathrm{PBN}$ radical adducts in maize roots after exposure to $1.0 \mathrm{mg} \mathrm{L}^{-1}$ DCPP enantiomers for 3 days: (a) PBN and DMSO without sample, (b) (S)-DCPP treatment, (c) (rac)-DCPP treatment, and (d) (R)-DCPP treatment.

that resulted in the upregulated POD was lower for $(R)$-DCPP $\left(0.02 \mathrm{mg} \mathrm{L}^{-1}\right)$ than $(\mathrm{rac})\left(0.05 \mathrm{mg} \mathrm{L}^{-1}\right)$ and $(S)\left(0.1 \mathrm{mg} \mathrm{L}^{-1}\right)$ enantiomers, indicating the existence of oxidative damage caused by $(r a c)$ - and ( $S$ )-DCPP exposures but less severe than the exposure of $(R)$-DCPP. Endogenous antioxidant enzymes, such as SOD and POD, play an important role in the repair of oxidative damage. ${ }^{29}$ However, a severe oxidative stress can inversely insult the enzyme to make them inactive. ${ }^{30}$ Therefore, inhibition of SOD and POD activities could be ascribed to the reason that, at higher DCPP concentrations, the increased enzyme activities were insufficient to scavenge excess ROS accumulated in plants, resulting in the loss of adaptive mechanisms. The evidence that SOD and POD activities were significantly upregulated by exposure to lower concentrations of $(R)$ DCPP than ( rac)- or ( $S$ )-DCPP demonstrates that (R)-DCPP can cause a stronger oxidative damage to maize than ( $\mathrm{rac}$ )- and (S)-DCPP.

Hydroxyl Radical Production Induced by DCPP Enantiomers. The hydroxyl radical $(\bullet \mathrm{OH})$ is the most reactive oxygen radical formed in living systems and plays a critical role in initiating and catalyzing a variety of radical reactions in the presence of oxygen. $^{31,32}$ Therefore, EPR analysis was further employed for direct determination of the $\bullet \mathrm{OH}$ formation in maize after exposure to DCPP. The spin trapping followed by EPR analysis provides direct determination of short-lived $\bullet \mathrm{OH}$, which can clearly manifest the degree of oxidative stress. ${ }^{33} \mathrm{~A}$ typical six-line (triplet of two lines in each) EPR spectrum of ROS and PBN adducts were observed in maize roots after exposure to DCPP (Figure 4, taking $1.0 \mathrm{mg} \mathrm{L}^{-1}$ as an example). The hyperfine coupling constants of the PBN adducts were $\alpha^{\mathrm{N}}=$ 14.9 G, $\alpha^{\mathrm{H}}=2.9 \mathrm{G}, \alpha^{\mathrm{N}}=16.2 \mathrm{G}, \alpha^{\mathrm{H}}=3.5 \mathrm{G}$, and $g=2.0062$, which correspond to that of the $\mathrm{PBN} /$ methoxy radical $\left(\bullet \mathrm{OCH}_{3}\right)$ and $\mathrm{PBN} /$ methyl radical $\left(\bullet \mathrm{CH}_{3}\right)$, respectively. ${ }^{34} \mathrm{PBN}$ itself was examined, and no EPR signal was observed (Figure 4). It was reported that $\bullet \mathrm{OH}$ reacted with DMSO to produce $\bullet \mathrm{CH}_{3}{ }^{35}$ Because oxygen cannot be completely secluded under anaerobic conditions, $\bullet \mathrm{CH}_{3}$ may be oxidized to $\bullet \mathrm{OCH}_{3} \cdot{ }^{36}$ Methyl gallate could scavenge $\bullet \mathrm{OH}$ but could not scavenge $\bullet \mathrm{CH}_{3} \cdot{ }^{35}$ When $0.2 \mathrm{~mL}$ of $1 \mathrm{M}$ methyl gallate was added to the homogenized plant tissue prior to the addition of PBN and DMSO, the signals of PBN adducts were totally inhibited (data not shown). Consequently, the signal of ROS captured in this study was recognized as $\bullet \mathrm{OH}$. 


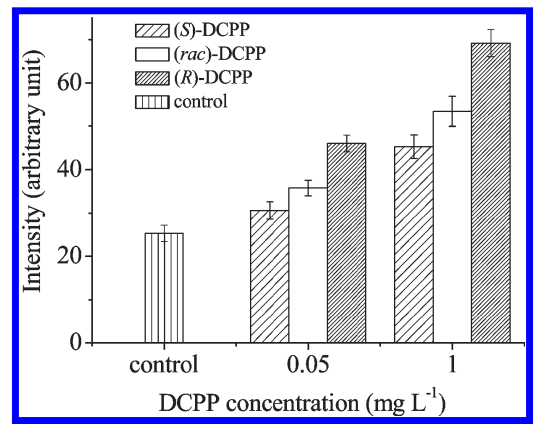

Figure 5. Radical intensity of $\mathrm{PBN}$ adduct EPR spectrum determined in maize roots after 3 days of exposure to 0.05 and $1.0 \mathrm{mg} \mathrm{L}^{-1}(S)$-, $(\mathrm{rac})$-, and (R)-DCPP. Differences between different concentrations and between different enantiomers were all significant at $p<0.05$ (mark symbol not shown).

The intensity of the EPR signal represents the $\bullet \mathrm{OH}$ level, and the $\bullet \mathrm{OH}$ intensity was calculated by the signal intensity of the second couple of the triplet and shown in Figure 5. The $\bullet \mathrm{OH}$ level was higher in roots of maize exposed to a higher concentration of DCPP. For treatment with different enantiomers, the intensity followed the order of $(R)$-DCPP $>(\mathrm{rac})$-DCPP $>(S)$ DCPP treatments. The highest $\bullet \mathrm{OH}$ level $(270 \%$ of the control) was obtained in the $(R)$-DCPP treatment at the concentration of $1.0 \mathrm{mg} \mathrm{L}^{-1}$, which was 1.5 and 1.3 times higher than $(S)$ - and (rac)-DCPP treatments, respectively. This result provides solicited evidence of the different oxidative damage of DCPP enantiomers to maize, with $(R)$-DCPP being the most serious. To our knowledge, this is the first time EPR analysis was employed to directly observe the formation of free radicals to distinguish enantioselective oxidative damage of chiral chemicals between enantiomers.

\section{AUTHOR INFORMATION}

\section{Corresponding Author}

*Telephone: 010-62849683. Fax: 010-62923563. E-mail: szzhang@rcees.ac.cn.

\section{Funding Sources}

This work was funded by the National Basic Research Program of China (Project 2009CB421603) and the National Natural Science Foundation of China (Projects 40730740 and 20921063).

\section{REFERENCES}

(1) Vetter, W. Enantioselective fate of chiral chlorinated hydrocarbons and their metabolites in environmental samples. Food Rev. Int. 2001, 17, 113-182.

(2) Liu, W. P.; Gan, J. Y.; Schlenk, D.; Jury, W. A. Enantioselectivity in environmental safety of current chiral insecticides. Proc. Natl. Acad. Sci. U.S.A. 2005, 102, 701-706.

(3) Ali, I.; Aboul-Enein, H. Y.; Ghanem, A. Enantioselective toxicity and carcinogenesis. Curr. Pharm. Anal. 2005, 1, 109-125.

(4) Liu, W. P.; Jing, Y.; Jin, M. Q. Enantioselective phytoeffects of chiral pesticides. I. Agric. Food Chem. 2009, 57, 2087-2095.

(5) Lenton, J. R.; Appleford, N. E. J.; Temple-Smith, K. E. Growth retardant activity of paclobutrazol enantiomers in wheat seedlings. Plant Growth Regul. 1994, 15, 281-291.

(6) Omokawa, H.; Kawata, Y.; Konnai, M. Interaction between optical isomerism and plant pharmacological action, change of modes of action and chirality of 1-( $\alpha$-methylbenzyl)-3-p-tolylurea. Pestic. Sci. 1993, 37, 107-112.

(7) Omokawa, H.; Murata, H.; Kobayashi, S. Chiral response of Oryzae and Paniceae plants in $\alpha$-methylbenzyl-3-p-tolylurea agar medium. Pest Manage. Sci. 2003, 60, 59-64.

(8) Tanaka, C.; Itagaki, M.; Arai, S.; Omokawa, H. Triceae plants preferentially responded to $S$-1- $\alpha$-methylbenzyl-3-p-tolylurea in root growth and tetrazolium reduction assays. Weed Biol. Manage. 2005, 5, 62-68.

(9) Kazuhiro, I.; Hisahiro, K.; Takako, N.; Hiroyoshi, O.; Hideyuki, T. Chiral effects of $(R)-/(S)-1$-( $\alpha$-methylbenzyl)-3-( $p$-tolyl)urea on the free amino acid levels in the root tips of rice and wheat. Weed Biol. Manage. 2009, 9, 87-92.

(10) Shimabukuro, R. H.; Hoffer, B. L. Enantiomers of diclofopmethyl and their role in herbicide mechanism of action. Pestic. Biochem. Physiol. 1995, 51, 68-82.

(11) Qian, H. F.; Hu, H. J.; Mao, Y. Y.; Ma, J.; Zhang, A. P.; Liu, W. P.; Fu, Z. W. Enantioselective phytotoxicity of the herbicide imazethapyr in rice. Chemosphere 2009, 76, 885-892.

(12) Ye, J.; Zhang, Q.; Zhang, A. P.; Wen, Y. Z.; Liu, W. P. Enantioselective effects of chiral herbicide diclofop acid on rice Xiushui 63 seedlings. Bull. Environ. Contam. Toxicol. 2009, 83, 85-91.

(13) Salin, M. L. Toxic oxygen species and protective systems of the chloroplast. Phvsiol. Plant. 1988, 72, 681-689.

(14) Scandalios, J. G. Oxygen stress and superoxide dismutases. Plant Phvsiol. 1993, 101, 7-12.

(15) Sun, Y. Y.; Yin, Y.; Zhang, J. F.; Yu, H. X.; Wang, X. R.; Wu, J. C.; Xue, Y. Q. Hydroxyl radical generation and oxidative stress in Carassius auratus liver, exposed to pyrene. Ecotoxicol. Environ. Saf. 2008, 71, 446-453.

(16) Wang, M. E.; Zhou, Q. X. Effects of herbicide chlorimuronethyl on physiological mechanisms in wheat (Triticum aestivum). Ecotoxicol. Environ. Saf. 2006, 64, 190-197.

(17) Song, N. H.; Yin, X. L.; Chen, G. F.; Yang, H. Biological responses of wheat (Triticum aestivum) plants to the herbicide chlorotoluron in soils. Chemosphere 2007, 68, 1779-1787.

(18) Contardo-Jara, V.; Wiegand, C. Biotransformation and antioxidant enzymes of Lumbriculus variegates as biomarkers of contaminated sediment exposure. Chemosphere 2008, 70, 1879-1888.

(19) Müller, M. D.; Buser, H. R. Conversion reactions of various phenoxyalkanoic acid herbicides in soil. 1. Enantiomerization and enantioselective degradation of the chiral 2-phenoxypropionic acid herbicides. Environ. Sci. Technol. 1997, 31, 1953-1959.

(20) Schneiderheinze, J. M.; Armstrong, D. W.; Berthod, A. Plant and soil enantioselective biodegradation of racemic phenoxyalkanoic herbicides. Chirality 1999, 11, 330-337.

(21) Bradford, M. M. A rapid and sensitive method for quantitation of microgram quantities of protein utilizing principle of protein-dye binding. Anal. Biochem. 1976, 72, 248-254.

(22) Beauchamp, C.; Fridovich, I. Superoxide dismutase: Improved assays and an assay applicable to acrylamide gels. Anal. Biochem. 1971, 44, 276-287.

(23) Lagrimini, L. M. Wound-induced deposition of polyphenols in transgenic plants overexpressing peroxidase. Plant Physiol. 1991, 96, 577-583.

(24) Shi, H. H.; Wang, X. R.; Luo, Y.; Su, Y. Electron paramagnetic resonance evidence of hydroxyl radical generation and oxidative damage induced by tetrabromobisphenol A in Carassius auratus. Aquat. Toxicol. 2005, 74, 365-371.

(25) Ma, Z.; Bielenberg, D. G.; Brown, K. M.; Lynch, J. P. Regulation of root hair density by phosphorus availability in Arabidopsis thaliana. Plant, Cell Environ. 2001, 24, 459-467.

(26) Zhou, Q. Y.; Xu, C.; Zhang, Y. S.; Liu, W. P. Enantioselectivity in the phytotoxicity of herbicide imazethapyr. J. Agric. Food Chem. 2009, $57,1624-1631$.

(27) Bowler, C.; Montagu, M. V.; Inzé, D. Superoxide dismutase and stress tolerance. Annu. Rev. Plant Physiol. Plant Mol. Biol. 1992, 43, 83-116. 
(28) Alscher, R. G.; Hess, J. L. Antioxidants in Higher Plants; CRC Press: Boca Raton, FL, 1993.

(29) Liu, X. L.; Zhang, S. Z.; Shan, X. Q.; Christie, P. Combined toxicity of cadmium and arsenate to wheat seedlings and plant uptake and antioxidative enzyme responses to cadmium and arsenate cocontamination. Ecotoxicol. Environ. Saf. 2007, 68, 305-313.

(30) Sun, Y. Y.; Guo, H. Y.; Yu, H. X.; Wang, X. R.; Wu, J. C.; Xue, Y. Q. Bioaccumulation and physiological effects of tetrabromobisphenol A in coontail Ceratophyllum demersum L. Chemosphere 2008, 70, $1787-1795$.

(31) Livingstone, D. R. Contaminant-stimulated reactive oxygen species production and oxidative damage in aquatic organisms. Mar. Pollut. Bull. 2001, 42, 656-666.

(32) Svend, J. K. J. Oxidative stress and free radicals. J. Mol. Struct. 2003, 666-667, 387-392.

(33) Li, F. Y.; Ji, L. L.; Luo, Y.; Oh, K. Hydroxyl radical generation and oxidative stress in Carassius auratus liver as affected by 2,4,6trichlorophenol. Chemosphere 2007, 67, 13-19.

(34) Burkitt, M. J.; Mason, R. P. Direct evidence for in vivo hydroxyl radical generation in experimental iron overload: an ESR spin-trapping investigation. Proc. Natl. Acad. Sci. U.S.A. 1991, 88, 8440-8444.

(35) Takeshita, K.; Fujii, K.; Anzai, K.; Ozawa, T. In vivo monitoring of hydroxyl radical generation caused by $\mathrm{X}$-ray irradiation of rats using the spin trapping/EPR technique. Free Radical Biol. Med. 2004, 36, 1134-1143.

(36) Yin, Y.; Jia, H. X.; Sun, Y. Y. Bioaccumulation and ROS generation in liver of Carassius auratus, exposed to phenanthrene. Comp. Biochem. Physiol., C 2007, 145, 288-293. 